\title{
Baby steps in E-Health: Internet of Things in a doctor's office
}

\author{
Jorge Oliveira e Sá1, João Cacho Sá2, Catarina Cacho Sá3, Manuel Monteiro ${ }^{4}$ and José \\ Luís Pereira ${ }^{1}$ \\ ${ }^{1}$ ALGORITMI Research Centre, University of Minho, Guimarães, Portugal \\ ${ }^{2}$ MiEEC, Faculty of Engineering of the University of Porto, Porto, Portugal \\ ${ }^{3}$ MiEGI, Engineering School of the University of Minho, Guimarães, Portugal \\ ${ }^{4}$ Hospital Particular São Lucas, Lagoa, Açores, Portugal \\ jos@dsi.uminho.pt; up201506676@fe.up.pt; a80519@alunos.uminho.pt; \\ manuel.monteiro@hpsl.pt; jlmp@dsi.uminho.pt
}

\begin{abstract}
IoT is a very common buzzword in e-Health because the creation of new and better healthcare services are possible through technology-push. There are great expectations in using IoT in healthcare, but most studies describe very futuristic and disruptive solutions for the healthcare real world. In a near future these solutions could be implemented, but now we need some "baby steps" to reach an e-Health with IoT technology. In this paper it is presented a solution to transform a doctor's office in an IoT office by helping the doctor attend a patien. This solution has impact in the doctor's work because (s)he has a limited time to attend a patient and a big part of this time is spent writing the results of the appointment in a computer record - the electronic patient record. By other hand the patient improve their experience because the doctor will give them more attention and care.
\end{abstract}

Keywords: m-Health $\cdot$ Clinical appointment $\cdot$ Patient-orientation $\cdot$ Bluetooth Low Energy · Connectivity · Electronic Patient Record

\section{Introduction}

IoT will be the engine for many paradigm shifts in the near future as it will require organizations to prepare meaningfully in terms of technological infrastructure, process optimization, change management and risk assessment. It will be the technological wave with the greatest impact in the world after the emergence of the Internet, transversal to all sectors of activity. It will bring new professions, new business and significant improvement in the quality of life of populations, but will also raise concerns in the areas of data security and privacy.

Today we can easily perceive the importance that the Internet has had in the society, namely in the way we communicate and the impact it has had in sectors such as education, banking, insurance, commerce, entertainment or the democratization of access to information. The Internet, coupled with the proliferation of mobile devices, has boosted the world's "appization" by offering features in a device that initially 
served to make and reject telephone calls to a multifunction device in which there is virtually no limit to its use.

Smartphones, and more recently smartwatches, and the endless fitness devices, allow heart rate monitoring and geo-referencing, among other indicators. Monitoring of heart rate, for later sharing of data on specialized platforms, is a good example of the $\mathrm{m}$-Health concept (term used for the practice of medicine and public health supported by mobile devices). m-Health change the way we interact with health care providers as patients are expected to show healthcare professionals the "collection" of data they regularly make with the help of mobile devices, such as blood pressure. However, the user sending such data to the health care unit (public health center or public hospital) in an automated manner and monitored by the health care unit automatically is not yet possible.

The potential of using IoT may, in the near future, lead to the creation of innovations, some of them totally disruptive in the health area.

Thus, this paper intends, through baby steps, i.e, not disruptive fashion, to describe the use of IoT in a doctor's office, a real environment.

The case described is in the context of a medical appointment that may occur at a Portuguese health center or hospital. Nowadays, the average duration of a medical appointment is twenty-one minutes, but, recently, there are some political recommendations to reduce this duration to fifteen minutes. Thus, a doctor may be seen as a clerk with accountable functions, like control the appointment duration and fill some computers screens with data collected in the appointment, than somebody interested to listen to patients, do correct clinical exams, or prescribe and explain the correct medications to patients.

In this context, the next section will explain the IoT concept. The section three will explain the evolution of m-Health to e-Health through IoT technologies. The section four, will describe the use of IoT technology in a doctor's office. The section five will present the conclusions.

\section{IoT Concept}

Although IoT is a recent buzzword, it was nicknamed this way for the first time in 1999 by [1]. However, the concept was developed many years earlier, in the early 1980s, by a group of programmers at Carnegie Melon University who modified a Coca-Cola machine by linking it to the Internet to obtain information on availability of the drink and the time the bottle drink was in the machine to ensure that the beverage was chilled and thus prevent them from moving in vain [2]. It is a simple example, but it explains the concept.

[1] refer that "today computers - and, therefore, the Internet - are almost wholly dependent on human beings for information. Nearly all the roughly 50 petabytes (a petabyte is 1,024 terabytes) of data available on the Internet were first captured and created by human beings - by typing pressing a record button, taking a digital picture or scanning a bar code. Conventional diagrams of the Internet include servers and routers and so on, but they leave out the most numerous and important routers of all: people. The problem is, people have limited time, attention and accuracy - all of which means they are not very good at capturing data about things in the real world". 
There are many types of sensors, many of them wireless and low cost that allow measuring and analyzing almost all kinds of things. The proliferation of these sensors, the improvement of wireless communications and the existence of identification technologies such as Radio Frequency Identification (RFID) or Near Field Communication (NFC) allows to connect all sorts of things to the Internet.

Therefore, it is possible to see the development of IoT in two perspectives: the technology and the coverage [3]:

- Technology - the main technology of IoT is the Internet, by providing a "free" platform to connect things and sharing intelligence and information turns the world with physical boundaries of nations to a "global village". Other relevant technology is RFID which enable linking things to Internet. Finally, the technology which enables machine to machine (M2M) computing is also relevant to IoT.

- Coverage - IoT phenomenon has evolved from only covering technology used by organizations to involving devices that affect common users' life.

\section{IoT to Health}

$\mathrm{m}$-Health is defined as "mobile computing, medical sensor, and communications technologies for health care" [4]. m-Health must be seen as a new way to understand healthcare services in three perspectives: [5]

1. m-Health simplifies the access to traditional and new services, because mobile devices services can be accessed everywhere and anytime;

2. m-Health is patient-oriented, i.e., the patients in most cases are responsible for the m-Health service; and

3. m-Health is personalized, the m-Health services are customized to fit the needs of patients.

m-Health is an evolutionary concept that provides both mobility and functionalities for different healthcare applications and it is a sub-segment of e-Health. Thus, e-Health covers Wireless Health (integration of wireless technology into traditional medicine), m-Health and Telehealth (for instance when two health professionals discussed a case by telephone or video-conference, or a video call between a patient and practitioner. Telehealth is an expansion of telemedicine because, telehealth includes prevention instead of clinical treatment).

e-Health describes any healthcare practice supported by an electronic information processing and communication, so it has broader reach than m-Health, see Fig. 1. e-Health has an important requirement that is the delivery of health services at anywhere and anytime, this requirement of "anywhere and anytime" means, that it is necessary to transmit messages between people (e.g.: patients, doctors, ...) independently of their location [6].

The IoT role in e-Health care allows the development of new technologies, some of which will be very disruptive, and enables interoperability (integration and intercommunication) between several "things" through the Internet. However, there is an issue because the use of these technologies is limited by several factors. The most important factor is that the data produced with IoT patient health devices needs to be sent to a health care unit (health care center or hospital) in an automated manner and 
monitored in the health care unit, and this is not yet occurring. This factor can be explained because the health care unit service is not ready to use these data and the doctors do not have time to analyze it. Other reasons are legal issues related to the privacy and security of the data transmitted.

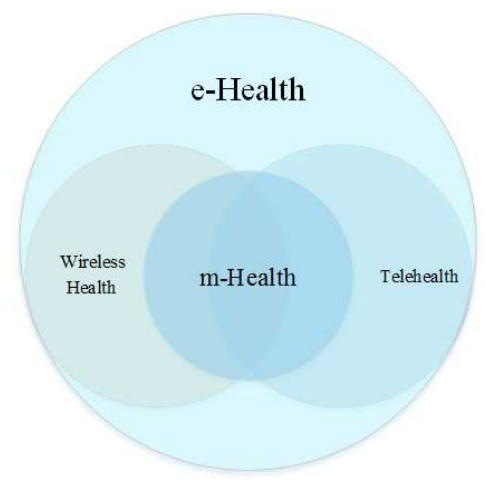

Fig. 1. e-Health, m-Health, Wireless Health and Telehealth

\section{$4 \quad$ IoT in a Doctor's Office}

\subsection{As-is Doctor's Office}

In a routine doctor's appointment the doctor measures, among other things, patient weight, height, blood pressure, pulse and temperature. In Fig. 2 it is represented an asis doctor's office and an as-is weighing scale. This mechanical physician weighing scale works by adjusting firstly the large sliding poise and secondly the small sliding poise. This operation requires time and operating skills.

As depicted in Fig. 2 the doctor must get up, walk to the device (red line path) and wait for the patient to do so. As the patient stands in the scale (s)he must try to find the right weight by adjusting the weighing device by sliding the poises. Then, the doctor must memorize the value acquired from the scale so (s)he can get back (again the red line path) to his desk and introduce the information by hand on the patient electronic record in the computer.
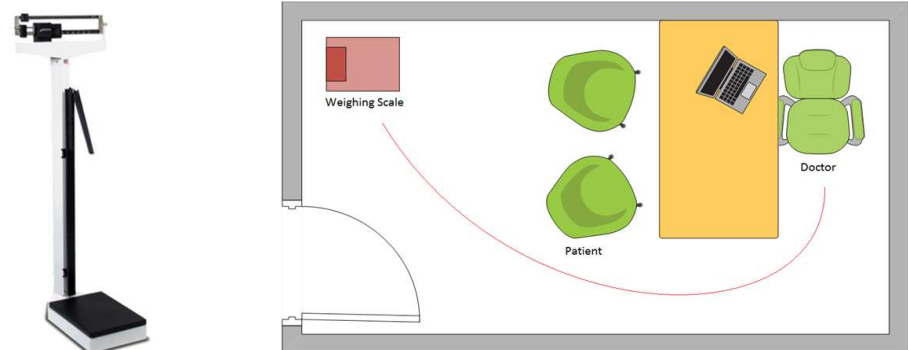

Fig. 2. As-is Weighing Scale and Doctor Office 
The as-is case has various problems. The doctor spends a lot of time in movement inside the office, and because of it, he can forget the values measured, there can be mistakes when the doctor introduces the values in the system and there is a lot of time wasted introducing the values in the system that could be used attending the patient.

\subsection{To be Doctor's Office}

Although there are domestic devices already in the market, see Fig. 3, that are able to be a IoT health care device, they are not ready for an intensive use of around 32 daily measurements. Therefore, these devices need to be improved to be used in a doctor's office.
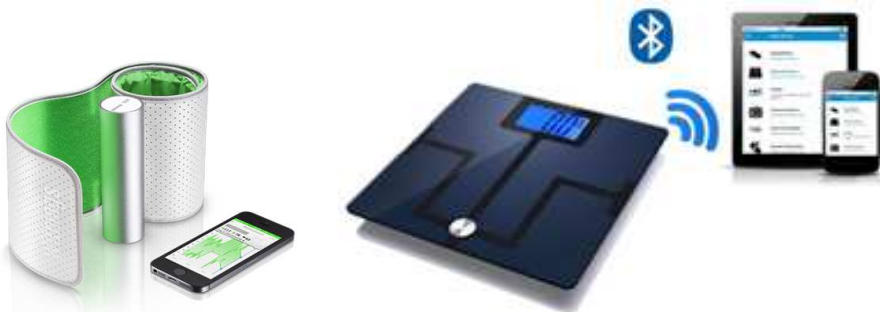

Fig. 3. Domestic IoT health devices, retrieved from $[7,8]$

Using again the weighing scale as an example, it features Bluetooth Low Energy (BLE) and has a battery life of 6 months and connects with a mobile application [9]. These specifications are also applicable to other domestic grade IoT devices. Fig. 4 will example a possible solution.
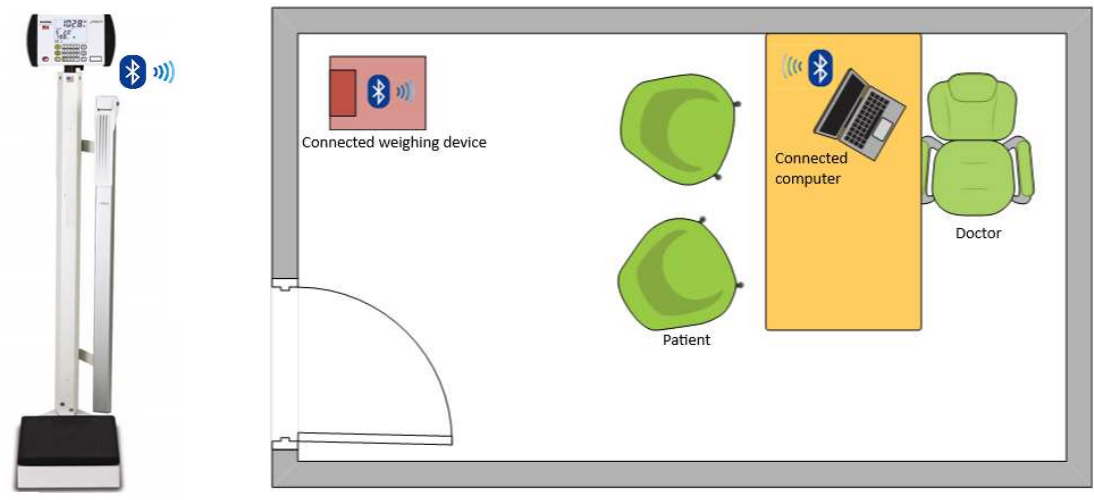

Fig. 4. To-be Weighing Scale and Doctor Office

In this doctor's office the devices are all connected by BLE with the office's computer, so it is a closed circuit. BLE, also known as Bluetooth Smart, operates at $2.4 \mathrm{GHz}$ and has a low power consumption, which means it can operate in coin size 
cells for months, it is small sized and cheap to manufacture and has compatibility with a large variety of devices.

The architecture presented in Fig. 5 purpose is to collect automatically the information from the devices and introduce it into the patient electronic record. From left side of Fig. 5 there are IoT devices like weighing scale, thermometer and other devices connected to a master device (left rounded blue in Fig. 5). This master device is connected to the computer doctor's office (round blue in the middle of Fig. 5). The computer doctor's office, in turn is connected to the patient electronic record (right side of Fig. 5), this electronic patient record may be in the Internet (Cloud).

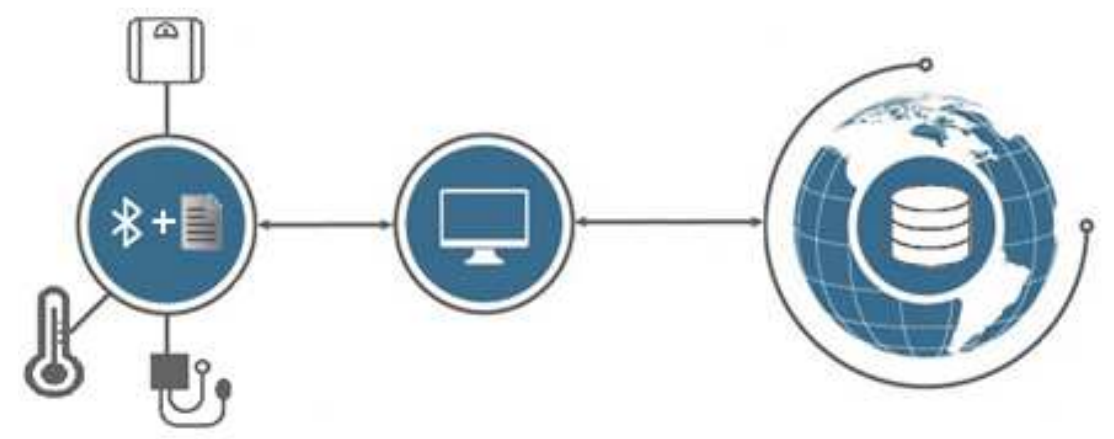

Fig. 5. Doctor's Office IoT Architecture

By connecting through BLE, the system is secure and connects all the medical devices as slaves to a master device in a unidirectional way but the master is connected to the office's computer in a bidirectional channel so it can trade information about the appointment. The master device may be a single board computer that has Bluetooth connectivity and $\operatorname{logs}$ the doctor and patient information and the data from the devices and, when requested, sends it to the doctor's computer. Using a master device makes it easier to connect the doctor's computer with the devices in an office and, by storing a log, makes the data recoverable if lost. It also makes the system more secure by only allowing the pre-defined slaves to connect to the master and only one computer for bidirectional communication, which can be authenticated by a password or pin.

The purpose of the $\log$ is to store the data during the appointment, in example, when the patient stands in the weighing scale the data is stored in the master device's $\log$, so that at the end of the appointment, or whenever needed, the doctor can auto-fill some fields of the electronic patient record.

The patient electronic record can then be filled by the doctor and sent to the central database, i.e., the patient electronic record central database.

\section{Conclusions}

This paper proposed an example solution of use of IoT in a Doctor's office. Our intention is not to be technology or service disruptive but present a viable solution with 
actual IoT technologies. Our solution has a real impact in the way the doctors perform a medical appointment, because the doctor has more time to attend the patient and give more attention to the clinical act, than to be clicking on keyboard. The patients will have a positive experience because the doctor will give them more attention and care.

This solution is a baby step into an IoT e-Health solution, because it is restricted to a doctor's office and not include any fancy sensor or wearable to be used by the patient. Thus, this solution does not raise very relevant data security concerns and communications between the IoT devices connected together are simple.

As said above, this is a baby step, but it is an important step to be done. Usually $\mathrm{m}$-Health and e-Health propose brilliant and disruptive solutions, but some are not yet possible to be implemented. Thus, in the healthcare area it is needed some small improvements, relatively easy to implement, with low cost and with possible big returns.

In the future, it will be relevant to implement the concept of IoT to other areas of public health care centers or public hospitals. Not only to implement solutions in e-Health, but to improve the experience of patients and practitioners, for example, it will be interesting to know the location of the patient in a hospital and send messages to the patient about the service waiting time and perhaps redirecting the patient to other service with lower waiting time.

Acknowledgments This work has been supported by COMPETE: POCI-01-0145FEDER-007043 and FCT (Fundação para a Ciência e Tecnologia) within the Project Scope: UID/CEC/00319/2013 and project QVida+: Estimação Contínua de Qualidade de Vida para Auxílio Eficaz à Decisão Clínica, NORTE-01-0247-FEDER-003446, supported by Norte Portugal Regional Operational Programme (NORTE 2020), under the PORTUGAL 2020 Partnership Agreement, through the European Regional Development Fund (ERDF).

\section{References}

1. Ashton, K., That 'internet of things' thing, RFiD Journal, vol. 22, $\mathrm{n}^{\circ}$ 7, pp. 97-114, 2009.

2. Coke Machine, 1998, retrieved 2-12-2016 from http://www.cs.cmu.edu/ coke

3. Jun, Z., Simplot-Ryl, D., Bisdikian, C., and Mouftah, H. T., The internet of things. IEEE Commun. Mag, vol. 49, no 11, pp.30-31, 2011.

4. Sundaravadivel, P., Mohanty, S., Kougianos, E., and Albalawi, U., An energy efficient sensor for thyroid monitoring through the IoT, 17th Int. Conf. on Thermal, Mechanical and MultiPhysics Simulation and Experiments in Microelectronics and Microsystems. IEEE, 2016.

5. Solanas, A., Martínez-Ballesté, A., and Mateo-Sanz, M., Distributed architecture with double-phase microaggregation for the private sharing of biomedical data in mobile health. IEEE Transactions on Information Forensics and Security, vol. 8, no 6, pp. 901-910, 2013.

6. Świątek, P., and Rucinski, A., IoT as a service system for eHealth. In IEEE 15th International Conference on e-Health Networking, Applications \& Services, IEEE, pp. 81-84, 2013.

7. Withings, Wireless Blood Pressure Monitor, 2016, retrieved from http://www.withings.com/eu/en/products/blood-pressure-monitor? At 30/11/2016.

8. Scale. Scale Bluetooth, 2016, retrieved from https://www.cambridgenetwork.co.uk/ public/products/sscales.jpg at 30/11/2016.

9. Chang, K. H., Bluetooth: a viable solution for IoT? [Industry Perspectives]. IEEE Wireless Communications, vol. 21, no 6, pp. 6-7, 2014. 